\title{
Tackling misconceptions in geometrical optics
}

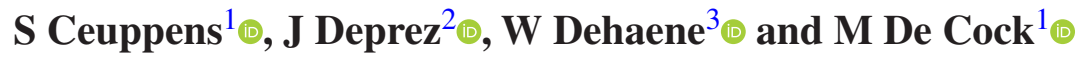 \\ ${ }^{1}$ Department of Physics and Astronomy, KU Leuven, Celestijnenlaan 200c box 2406, 3001 \\ Leuven, Belgium \\ ${ }^{2}$ Department of Mathematics, KU Leuven, Celestijnenlaan 200b box 2400, 3001 Leuven, \\ Belgium \\ ${ }^{3}$ ESAT-MICAS, KU Leuven, Kasteelpark Arenberg 10 box 2443, 3001 Leuven, Belgium \\ E-mail: stijn.ceuppens@kuleuven.be and mieke.decock@kuleuven.be
}

\begin{abstract}
To improve the teaching and learning materials for a curriculum it is important to incorporate the findings from educational research. In light of this, we present creative exercises and experiments to elicit, confront and resolve misconceptions in geometrical optics. Since ray diagrams can be both the cause and the solution for many misconceptions we focus strongly on improving understanding of this tool to solve and understand optical phenomena. Through a combination of a conceptual understanding programme (CUP) and provocative exercises with ray diagrams we aim to elicit conceptual or cognitive conflict and exploit this to tackle misconceptions and increase students' conceptual understanding through inquiry. We describe exercises for image formation by a plane mirror, image formation by a convex lens and indirect and direct observation of a real image formed by a convex lens as examples of our approach.
\end{abstract}

\section{Introduction}

Optical phenomena and technology are everywhere and their applications are only increasing. Therefore, it is not surprising to find geometrical optics in most science curricula and a topic of interest in physics education research (PER). Studies from PER have repeatedly shown that students have difficulties to understand concepts underlying optical phenomena both pre-instruction and post-instruction [1-11]. The preconceptions students construct before entering formal optics education are so entrenched in their reasoning that classical optics teaching does not always allow to change their ideas to more scientific ones. In order to have students change their ideas, they should be mentally activated in targeted student activities (e.g. [12]). The Flemish STEM@ school project aims to develop innovative learning materials on integrated STEM (science, technology, engineering and mathematics). One of the underlying principles in the design of these materials is taking into account findings from discipline based educational research. In this paper, we report how findings on particular student difficulties in optics informed the design of student activities to tackle misconceptions, using an inquiry based and cooperative learning approach.

\section{Student difficulties in geometrical optics}

\subsection{Image formation by a plane mirror}

Many students have difficulty explaining the image location of an object placed in front of a plane mirror. They state the image is formed 


\section{S Ceuppens et al}

on the mirror's surface or use a 'line-of-sight' argument to incorrectly state that the image of an object is located at the intersection of the mirror plane and the line-of-sight connecting the observer and the object, or at the point symmetrically behind the mirror but still on the line-ofsight $[1,3]$. Also, predicting whether the image of an object is visible from a given point of view proves to be difficult for both students and adults [4]. This relates to the effect of the point of view and the size of the mirror on the field of view. Many people incorrectly believe that plane mirrors have 'zooming capabilities': by moving closer you see less of yourself and by moving further away, you see more [1, 3, 10]. Additionally, Bianchi and Savardi have shown that about half of their adult respondents are strongly biased towards thinking that the field of view of a mirror is the orthogonal space in front or beyond it for eccentric viewpoints [4].

\subsection{Image formation by a convex lens}

Goldberg and McDermott [2] reported significant student difficulties with real image formation by a converging lens when students were presented with a simple setup of a light bulb, a converging lens and a screen and asked what would happen if half of the lens were covered. Before instruction, almost all respondents replied that half of the image would vanish. After instruction, this incorrect idea persisted with $55 \%-75 \%$ of the students, depending on the instruction received.

\subsection{Indirect and direct observation of a real image formed by a convex lens}

Goldberg and McDermott [2] also reported that many students - when given a simple setup of a luminous object and a converging lens-were unable to identify the full conditions to observe the image. Students think that a screen is necessary to see the image-i.e. indirect observationor that the observer's eye should be at the location of the screen, effectively replacing the screeni.e. direct observation. Most students did not know that the image could be observed directly by looking from anywhere in a well-defined space along the optical axis behind the lens.

\subsection{Ray diagrams}

Constructing a ray diagram is a powerful geometrical algorithm to depict phenomena that can be explained by geometrical optics principles in which a light ray is the key concept allowing to explain the rectilinear propagation of light and image formation. However, research has shown that students do not always recognize these rays as useful geometrical tools but tend to see them as physical entities $[2,13,14]$. A particular problem is that the rays/lines in a ray diagram do not always have a matching equivalent in reality which can create confusion about the interpretation of a diagram and its relation with what happens in reality. In a diagram with a plane mirror for instance, the rays can be extended behind the mirror to determine the image location, but there are no rays behind the mirror in reality since it is a virtual image (see section 3.1). Also the principal rays used to determine the location of an image formed by a converging lens are a geometrical tool since even if the matching ray in reality were blocked, the principal ray in the diagram can still be used to determine the image location (see section 3.2). Similarly with a diverging lens, the extensions of the rays help to determine the image location. To distinguish between the rays that exist in reality and those that do not have a matching equivalent, the former are drawn as full lines and the latter are drawn as dashed lines. This distinction is important and must be explicitly made by the teacher, ideally also illustrated with an experimental setup to show the presence and absence of light rays in which a laser can serve as a light ray. In addition, an insightful ray diagram contains only a sufficient number of well chosen rays and students tend to forget that the infinite number of other possible rays also contribute to the image formation. Lastly, the conditions to observe an image are often disconnected from ray diagrams. Students usually understand that a screen at the image location allows for indirect observation, but they often fail to understand how the image can be observed directly. These issues show that ray diagrams can cause student difficulties, especially when translating a real system into a ray diagram and vice versa. However, when used appropriately, ray diagrams offer opportunities to elicit conceptual conflicts and to resolve 
Tackling misconceptions in geometrical optics

misconceptions by applying them consistently in carefully designed exercises.

\section{Student activities to tackle misconceptions}

To tackle the described difficulties, we designed student activities using the 'elicit, confront and resolve' approach [12] combined with ideas from conceptual understanding program (CUP) [15], which are direct applications of inquiry and cooperative learning. We focus particularly on:

- The use of ray diagrams: in all exercises we scaffold students to use ray diagrams.

- The use of observer's eyes, which is twofold: firstly, they indicate the locations (along a line) from which a particular image point can be observed. Secondly, in diagrams containing lenses it makes clear that light rays do not end in the image point but continue until they are absorbed by an object or an eye. In our diagrams we always draw a single eye for each single ray. It is important to note that because of this, students might think that a single ray is sufficient to determine the location of the image point, which it is not. It is a very different task to determine the location of an image point or to determine the location from which an observation can be made. The former requires a minimum of two rays in the case of image formation by a lens and when using the physical method with plane mirrors (discussed in section 3.1) and the latter only requires a single one-using backwards selection of a path of light [13] — after the image point has been determined.

- The implementation of activating student activities: first, students learn the basics of ray diagrams; afterwards, they receive provocative exercises which confront them with apparently conflicting situations that challenge their understanding. In these exercises, students first solve a problem individually, then discuss their solutions in small groups and finally discuss with the whole class. When consensus is reached the teacher can confirm the answer or indicate potential conflicts in the students' reasoning and guide them to the correct answer through inquiry. Students are actively engaged and responsible for their own learning with minimal guidance from the teacher and they learn with and from their peers. By applying the same concepts and reasoning, reflecting on them and then generalizing them in a variety of such exercises, students can construct a coherent conceptual framework applicable across all contexts instead of memorizing specific solutions to specific problems and ending up with an incoherent conceptual framework [12].

To support these activities, teachers are provided with a list of tips and possible pitfalls, e.g.: draw arrows on the rays, draw the reflected or refracted rays through until they reach an observer's eye, draw regular rays in addition to principal rays, etc., which they can then make explicit to students. In the following sections we describe selected examples in which we apply this approach.

\subsection{Image formation by a plane mirror}

To determine the image formed by a plane mirror, students are taught two methods. Figure 1(a) shows the 'mathematical method' in which symmetric distances are measured with respect to the mirror's plane. This though does not provide much insight in the phenomenon of reflection, hence students are also taught the 'physical method': using ray diagrams and the law of reflection as shown in figure 1(b). Students are instructed to primarily use the latter. Note that a grid is absent in figure 1, since the presence of a grid might stimulate the use of the mathematical method and the absence of a grid might stimulate the use of the physical method. Additionally, in figure 1(b), each observer eye receives only a single ray since the situation depicted here is that of a point object. Teachers should be aware that students might interpret this in such a way that they conclude that only a single ray is necessary to determine the image point, which is incorrect since at least two are necessary in this method.

Students then work on problems evoking the earlier mentioned difficulties, using the described approach.

- In figures 2(a) and (b) students are asked to determine the image of objects which are not completely in the orthogonal space in front of the mirror or for which the mirror is 'too 
(a)

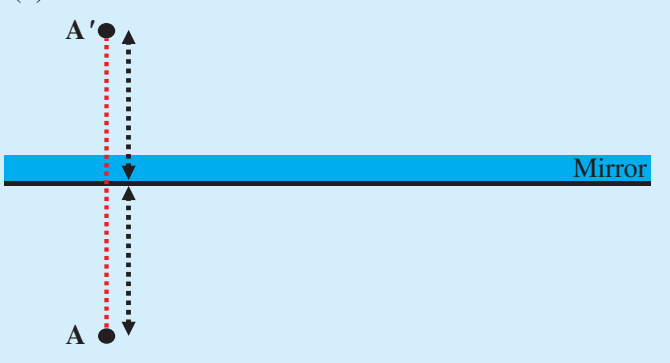

(b)

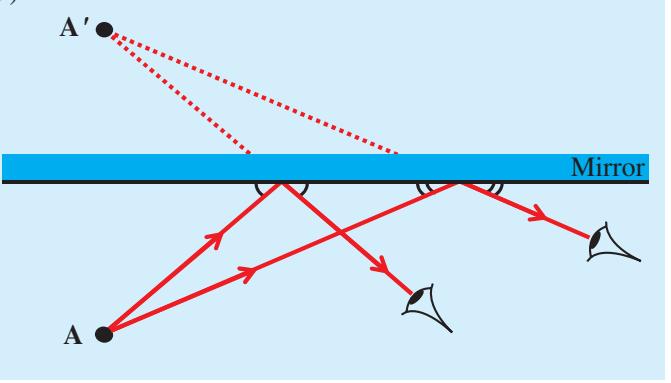

Figure 1. (a) Mathematical method: using equal distance with respect to the mirror plane to find image point A'. (b) Physical method: using the law of reflection through a ray diagram with equal angle of incidence and angle of reflection for two randomly chosen rays starting from object point A and ending in 'observer eyes'. The intersection of the extended reflection rays identifies image point A'.

(a)

Use a ray diagram to determine the image location of objects A and B.

Mirror (b)

Use a ray diagram to determine the image location of object A.

Mirror

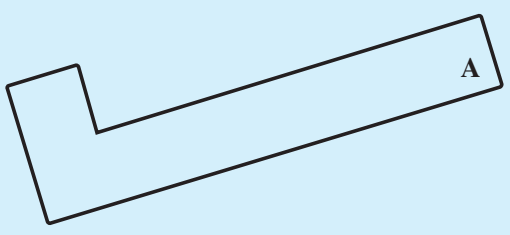

Figure 2. Ray diagram exercise on reflection at plane mirrors, abbreviated instructions are shown. Elicited issues: (a) and (b) some points are outside the perpendicular space of the mirror, the mirror is 'too small'.

small'. Using ray diagrams can help students understand that also the points outside the orthogonal space have an image and that determining the image of an object is not the same as determining the field of view of the mirror.

- The problem in figure 3 confronts students with the 'line-of-sight' argument $[1,2,16]$ by locating the image point for two different observers. By explicitly asking to use ray diagrams, they realise the image is independent of the observers' locations.

- In the 'reverse engineering problems' (figures 4(a) and (b)) students must apply known principles to non-standard situations: in these examples using the physical method in reverse to find the mirror's plane instead of the image. These two examples clarify the misconception that light rays are not emitted from the eyes in order to see [11], but that starting from the eyes can be a useful solution strategy. The exercise in figure 4(b) includes a catch-there is no realistic solution because one of the observers would be behind the mirror-which requires students to reflect on their solution and interpret the ray diagram back to the real world situation.

- The exercises in figures 5(a) and (b) show two examples which can help tackle the idea of a mirror's 'zooming capacities' and help to correctly determine the minimum mirror size to see the full image of a person in a mirror. Students are provided with multiple versions of this exercise in which e.g. the distance between the person and the mirror varies. Additionally, we encourage to use an interactive applet from 
Tackling misconceptions in geometrical optics

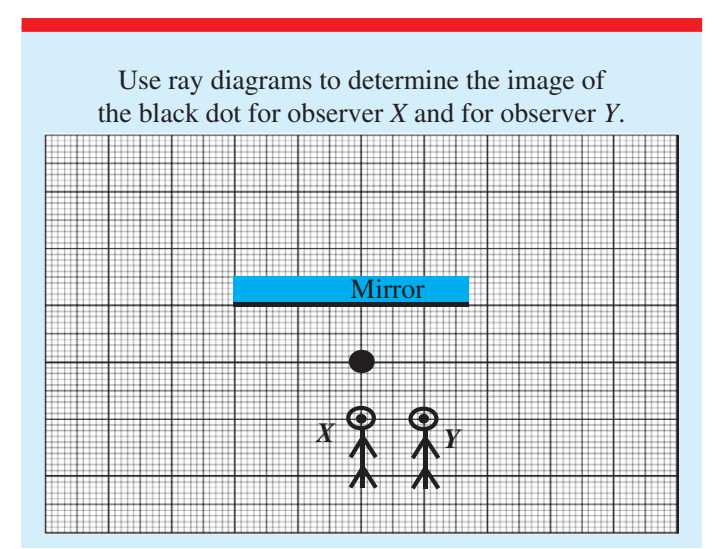

Figure 3. Ray diagram exercise on reflection at plane mirrors, abbreviated instructions are shown. Elicited issues: (a) line-of-sight argument, the image is observer dependent.

the Wolfram Demonstrations Project [17] or similar, which allows students to dynamically study the effect of mirror size and the distance to the mirror. Also, students carry out a simple experiment in which they use a plane mirror and a marker and indicate which part of their face they can see in the mirror and compare this for different object distances.

\subsection{Image formation by a convex lens}

To tackle the problem with the partially covered lens, Goldberg and McDermott [2] advise teacher demonstrations to clarify the effect of covering parts of the lens. Based on their work, we developed a more active tutorial in which students cooperate in groups of four. Students start with a typical situation with an object and a converging lens as shown in figure 6 .

- Students individually determine the image location. Each must do this differently: one has to use all principal rays, the other three each have to use a different pair of principal rays. They must also draw at least one 'regular' ray in a different color.

- Next, they draw a predetermined opaque block on their diagram — which 'blocks' the principal ray through $F_{2}$-and predict the effect on the image using a ray diagram. The situation in figure 7(a) shows what a student using the principal rays through $F_{1}$ and $O$ is likely to find, which is unlikely to cause any concern since no rays are 'blocked'. Figure 7(b) shows a possible situation of a modified diagram of a student using the principal rays going through $F_{1}$ and $F_{2}$ and intuitively-and incorrectly — believes that a partial image will be formed and tries to justify this by drawing the rays starting from the highest non-blocked object point. Figure 7(c) shows a correct diagram in which the principal rays through $O$ and $F_{2}$ are used and in which the latter one can still be used to determine the location despite the dashed part of the ray not existing in reality, i.e. the dashed part does not match a ray which exists in reality but is simply a geometrical tool to determine the image location. This diagram shows that the image is fully formed but still does not illustrate the entire effect on the image. Figure 7(d) shows a similar diagram as in figure 7(c) and additionally illustrates that a section of the light cone is blocked which decreases the amount of light contributing to the brightness of the image point, thus resulting in a fully formed but less bright image. This diagram-which students are unlikely to come up with themselves - is the one which should be understood at the end of this exercise, since it shows that the principal ray through $F_{2}$ can still be used and explains the effect on the image $V$ '.

- Students compare approaches and discuss till consensus. Each group draws its final diagram and compares with other groups. After a class consensus the teacher reveals the correct answer and discusses the incorrect ones. Throughout the tutorial we encourage to use of real optical artifacts (a luminous object, a converging lens and a screen) to better link the ray diagrams with the real world system.

- Besides 'blocked' principal rays, other issues can challenge students' understanding, e.g. not passing through the drawn lens. In figure 8(a) the lens seems to be 'too small' for the object and two principal rays will not intersect the lens.

- The exercise in figure 8 (b) relates to the completeness of the image. It shows an asymmetric or partial lens which might confuse students since regular exercises have lenses with their optical center in their geometrical center. This aims to clarify that even asymmetric/partial lenses produce a full 


\section{S Ceuppens et al}

(a)

Use a ray diagram to determine the location of the mirror plane.

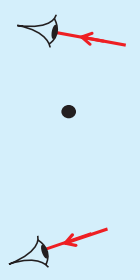

(b)

Use a ray diagram to determine the location of the mirror plane.
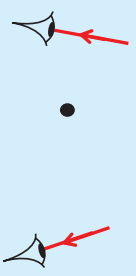

Figure 4. Ray diagram exercise on reflection at plane mirrors, abbreviated instructions are shown. Elicited issues: (a) and (b) reverse application of known principles, light must be emitted by the eyes to see an object/image.

(a)

1. Use a ray diagram to determine the image of the person.

2. Use a ray diagram to determine the field of view of the person. (How much of himself can the person see in the mirror?)

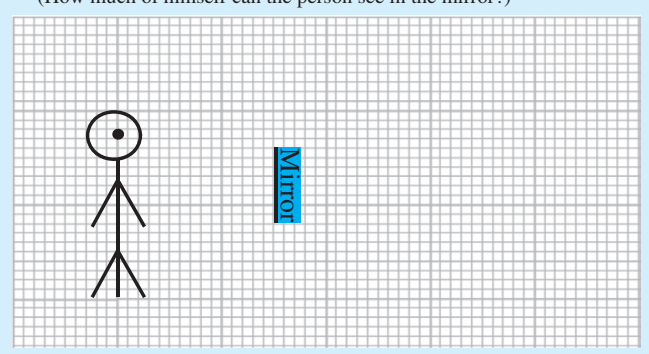

(b)

1. Use a ray diagram to determine the minimum size of the mirror so the person can see his full mirror image.

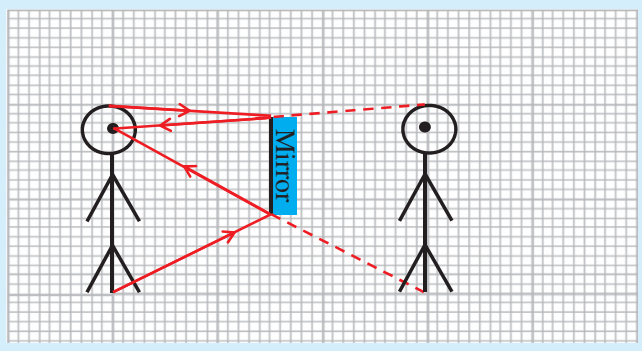

Figure 5. Ray diagram exercise on reflection at plane mirrors, abbreviated instructions are shown. Elicited issues: zooming capability of a mirror, dependency of object distance and mirror size; (a) exercise in which the mirror is not large enough to see the full image; (b) ray diagram to determine the minimum required mirror size to see the full image.

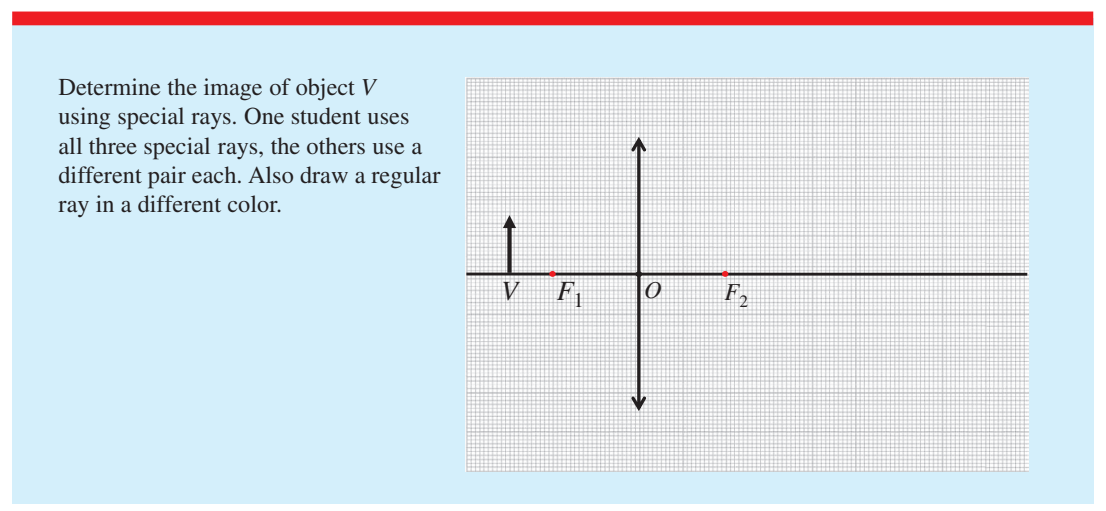

Figure 6. Standard situation with object $V$ and a convex lens with focal points $F_{1}$ and $F_{2}$ for which students must determine the image of $V$. 


\section{Tackling misconceptions in geometrical optics}

(a)

Consider the optical center $O$ as the origin of a coordinate system and draw an opaque block between coordinates $(-1,1)$ and $(-1,2)$. Explain the effect on image $V^{\prime}$ using a ray diagram.

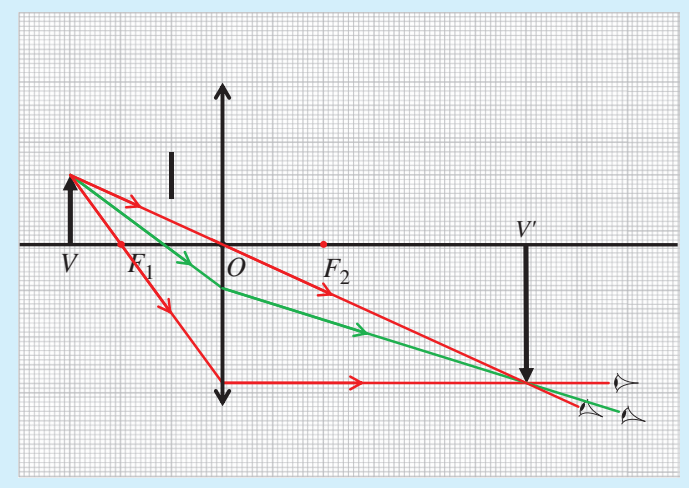

(c)

Consider the optical center $O$ as the origin of a coordinate system and draw an opaque block between coordinates $(-1,1)$ and $(-1,2)$ Explain the effect on image $V^{\prime}$ using a ray diagram.

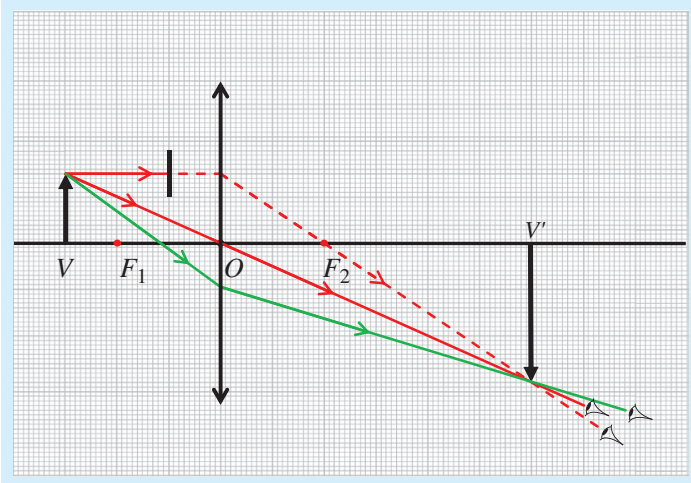

(b)

Consider the optical center $O$ as the origin of a coordinate system and draw an opaque block between coordinates $(-1,1)$ and $(-1,2)$. Explain the effect on image $V^{\prime}$ using a ray diagram.

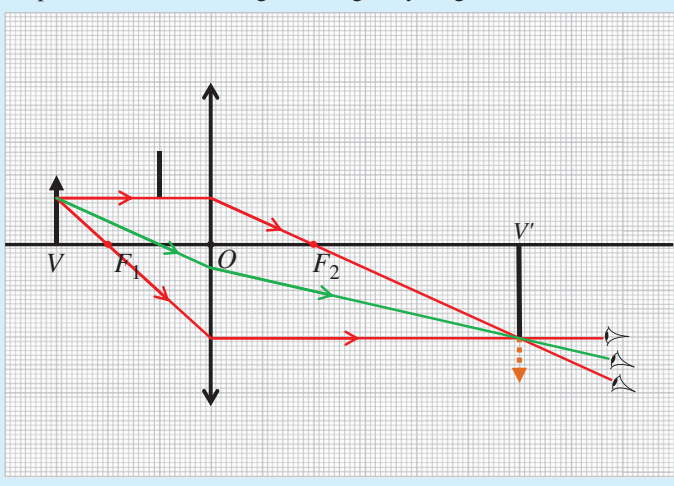

(d)

Consider the optical center $O$ as the origin of a coordinate system and draw an opaque block between coordinates $(-1,1)$ and $(-1,2)$ Explain the effect on image $V^{\prime}$ using a ray diagram.

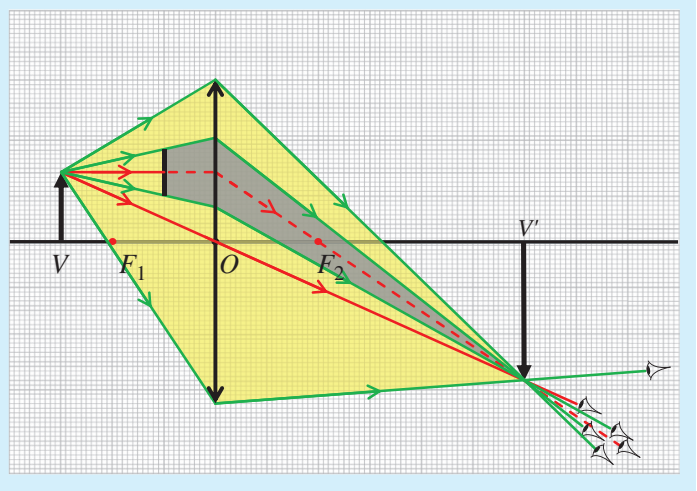

Figure 7. Possible outcomes with principal rays in red and regular rays in green. (a) Correct diagram without any 'blocked' rays, which does not create a conceptual conflict. (b) Incorrect diagram which attempts to explain the formation of a partial image by drawing two principals rays from the highest non-blocked point of the object thus creating the idea that the top (orange) part of the image is not formed; (c) correct diagram to determine the image location. Though the principal ray through $F_{2}$ is blocked in reality the dashed extension is still used to correctly determine the image location. (d) Correct ray diagram using the same principal rays as in (c) to determine the image location. In addition this diagram also illustrates the effect of the opaque block: the light cone which determines the brightness of the image point is shown and part of the cone is blocked, thus resulting in a less bright image point.

image if there are rays from all object points reaching a part of the lens and that the image can always be determined through consistent application of ray diagrams.

- Figure 9 shows a 'reverse engineering' problem in which students must determine the lens location and focal points by using the same ray diagram techniques as in regular exercises, but reverse the logic. We also ask students students to calculate the results using the lens formula, thus showing that both ray diagrams and formulae give the same results when applied and interpreted correctly.

\subsection{Indirect and direct observation of a real image formed by a convex lens}

Also to distinguish between direct and indirect observation and study their conditions, we use the same approach. 


\section{S Ceuppens et al}

(a)

Determine the image of object $V$ using special rays. Explain the effect of the small lens on the image.

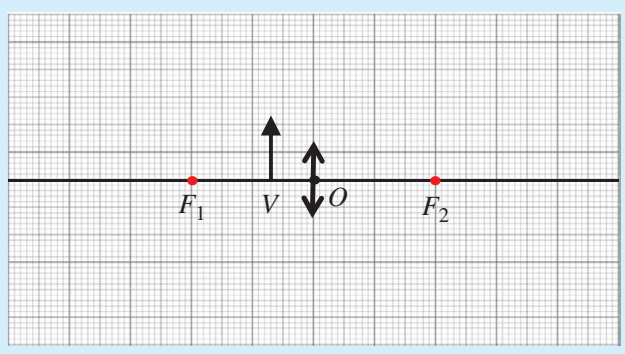

(b)

Determine the image of object $V$ using special rays. Explain the effect of the partial/asymmetric lens on the image.

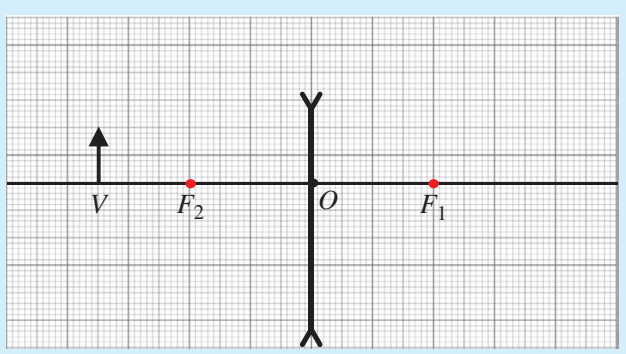

Figure 8. (a) A 'too small' lens with two principal rays not intersecting the drawn lens; (b) asymmetric or partial (convex) lens.

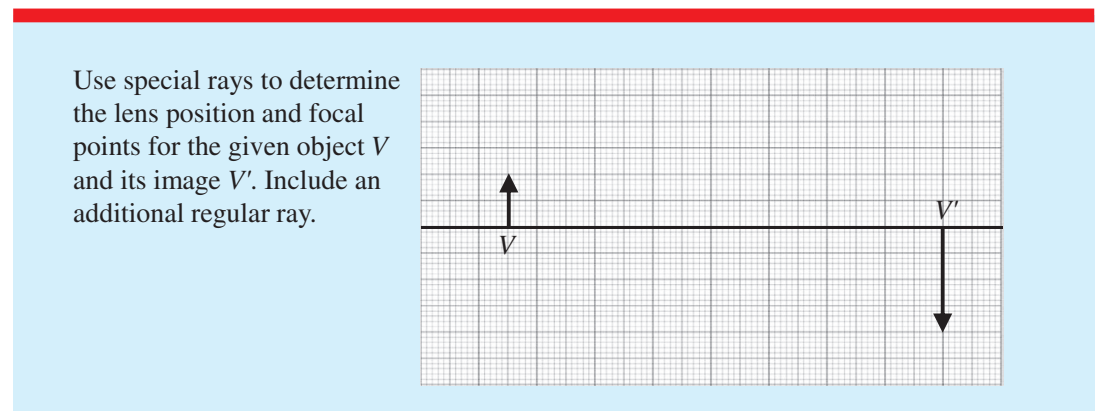

Figure 9. Reverse application of known principles to find the lens position and focal points.

- First students figure out how both observers can indirectly see the image using a screen and motivate this with a ray diagram. The goal is to end up with a diagram similar to figure $10(a)$.

- In a second step, they determine for which of the given observers the image is directly visible_-see figure 10(b)—and identify the area from which the full image is always directly observable. The conclusion should be that there is an area along the optical axis behind the lens which can be determined by drawing the rays that start at the end points of the object and pass through the end points of the lens as shown in figures $10(\mathrm{c})$ and (d). Note that these are regular rays, which aides in countering the idea that only principal rays contribute to the image formation. Another important point is that the rays do not stop at the image point but continue and can reach the eye of an observer. Next, the overlapping area which allows for the direct observation of the full image is identified.

- Finally, the diagrams for indirect and direct observations are compared and discussed.

\section{Discussion and conclusion}

A total of 30 secondary schools from across Flanders voluntarily implemented these activities during two consecutive years (2015-2017) in the 9th grade with a typical student's age of 14-15 and a typical boy/girl ratio of 1.5. All of the students participated in the STEM@ school project, meaning they opted for a STEMcurriculum with a strong science component. Before, in between and after teaching the curriculum, the teachers-who range from new to experienced-were invited to semi-structured meetings with their colleagues from the other STEM-disciplines and from other participating schools to discuss the implementation, share 
(a)

Determine the image of object $V$ using special rays; and show how the observation eyes can observe the image when a screen is placed at the image position.

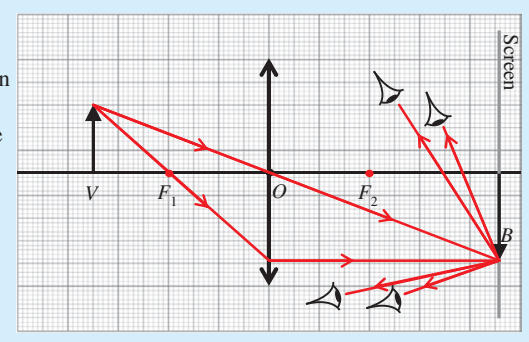

(b)

Use special rays and regular rays to determine which eye can directly observe the top of the image.

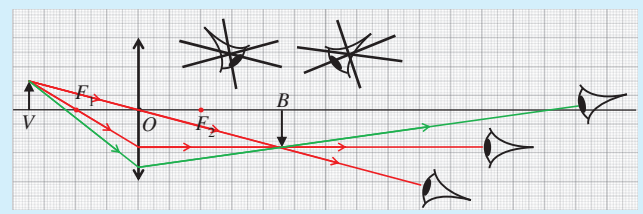

(d)

Use special rays and regular rays to determine which eye can directly observe the top of the image.

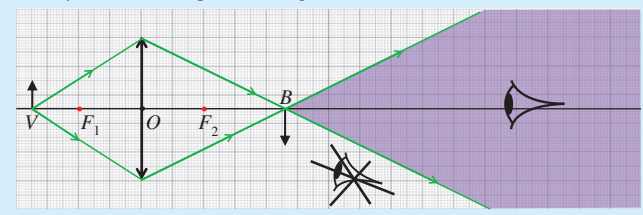

(c) Use special rays and regular rays to determine which eye can directly observe the top of the image.

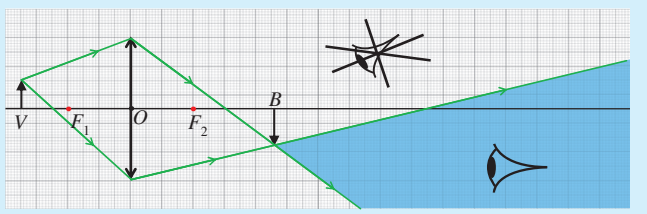

(e) Use special rays and regular rays to determine which eye can directly observe the top of the image.

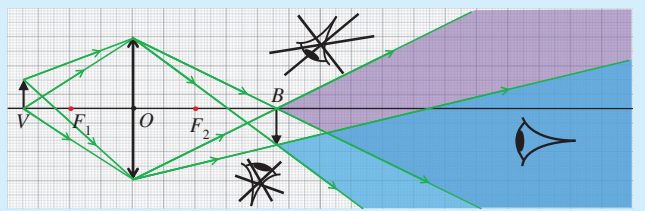

Figure 10. (a) Indirect observation by diffuse reflection; (b) direct observation from behind the lens and along the optical axis; (c)-(e) steps to determine the area from which the whole object can be observed directly.

their experiences and learn from each other. After the implementation, online questionnaires were administered to each team of teachers-one per school-to gather additional feedback for future improvements to the STEM-curriculum. The teachers' feedback and assessment of their students is based on the day-to-day interaction with their students and their own assessment tools (not-standardised across the various schools). This teacher feedback showed that they much appreciated the focus on misconceptions and that many found the exercises an improvement compared to classical textbooks. When queried about misconception retention after the course was taught, teachers' opinions were that low performing students likely still retain some misconceptions but that others have less or almost none as far as the teachers are aware. Furthermore, teachers reported increased students' enthusiasm and engagement for cooperation. These observations are in line with our goal to improve students' conceptual understanding through CUP and exercises with ray diagrams to provoke a conceptual conflict and elicit, confront and resolve misconceptions. Based on these positive experiences, we advise to include this approach in any new course design.

\section{Acknowledgments}

This research was funded by the Flemish IWTSBO project STEM@school.

\section{ORCID iDs}

S Ceuppens (D) https://orcid.org/0000-0002-59606539

J Deprez (1) https://orcid.org/0000-0003-47433631

W Dehaene (1) https://orcid.org/0000-0002-67927965

M De Cock (1) https://orcid.org/0000-0002-24891528 


\section{S Ceuppens et al}

Received 5 March 2018, in final form 9 May 2018

Accepted for publication 18 May 2018

https://doi.org/10.1088/1361-6552/aac604

\section{References}

[1] Goldberg F M and McDermott L C 1986 Student difficulties in understanding image formation by a plane mirror Phys. Teach 24 472-81

[2] Goldberg F M and McDermott L C 1987 An investigation of student understanding of the real image formed by a converging lens or concave mirror Am. J. Phys. 55 108-19

[3] Cummings K and Grillo E 2005 Conceptual underpinnings of students' ability to understand reflections from a plane mirror AIP Conf. Proc. 790 185-8

[4] Bianchi I and Savardi U 2012 What fits into a mirror: naïve beliefs about the field of view J. Exp. Psychol. 38 1144-58

[5] Tural G 2015 Cross-grade comparison of students' conceptual understanding with lenses in geometric optics Sci. Educ. Int. 26 325-43

[6] Kaltakci-Gurel D, Eryilmaz A and McDermott L C 2016 Identifying preservice physics teachers' misconceptions and conceptual difficulties about geometrical optics Eur. J. Phys. 37045705

[7] Anderson C W and Smith E L 1986 Children's conceptions of light and color: understanding the role of unseen rays Technical Report The Institute for Research on Teaching, East Lansing, Michigan

[8] Wosilait K, Heron P R L, Shaffer P S and McDermott L C 1998 Development and assessment of a research-based tutorial on light and shadow Am. J. Phys. 66 906-13

[9] Viennot L, Chauvet F, Colin P and Rebmann G 2005 Designing strategies and tools for teacher training: the role of critical details Examples Opt. 89 13-27

[10] Pompea S M, Dokter E F, Walker C E and Sparks R T 2007 Using misconceptions research in the design of optics instructional materials and teacher professional development programs Education and Training in Optics and Photonics (Optical Society of America) https://doi.org/10.1364/ ETOP.2007.EMC2

[11] Allen M 2010 Misconceptions in Primary Science 1st edn (Milton Keynes: Open University Press)

[12] McDermott L C 1993 Guest comment: how we teach and how students learn-a mismatch? Am. J. Phys. 61295

[13] Colin P and Viennot L 2001 Using two models in optics: students' difficulties and suggestions for teaching Am. J. Phys. 69 S36-44

[14] Santana A, Rodrguez Y and Gmez E A 2012 Construction of ray diagrams in geometrical optics: a media-focused approach Phys. Educ. 47715

[15] Mills D, McKittrick B, Mulhall P and Feteris S 1999 CUP: cooperative learning that works Teach. Phys. 34 11-6

[16] Favale F and Bondani M 2013 Misconceptions about optics: an effect of misleading explanations? Education and Training in Optics and Photonics ed F P C Martins Costa and M Zghal (Optical Society of America) https://doi.org/10.1117/12.2070520

[17] Vuilleumier B 2018 'Optics: law of reflection' from the wolfram demonstrations project http://demonstrations.wolfram.com/ OpticsLawOfReflection/ (Accessed: 12 February 2018)

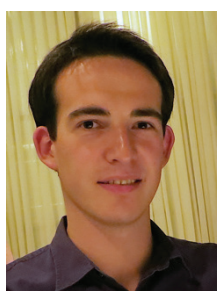

Stijn Ceuppens obtained his Master of Science in Physics at Universiteit Antwerpen in 2011 and his Teacher Degree in 2012 at the same university. $\mathrm{He}$ is a PhD student at KU Leuven and works on the development of integrated STEM course materials for secondary education in Flanders (Belgium) and on the close relation between physics and mathematics.

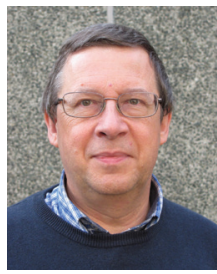

Johan Deprez obtained a PhD in Mathematics at KU Leuven in 1989. Currently, he is Associate Professor in the Mathematics Department at KU Leuven, where he is responsible for the Mathematics Teacher Education Program. One of his main research interests is the teaching and learning of mathematics in close interaction with other subjects.

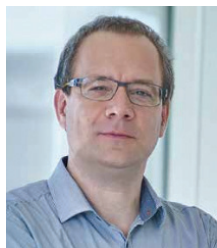

Wim Dehaene is a professor at KULeuven since 2002. Before that time he was working for Alcatel Microelectronics as a senior project director. He holds a PhD (1996) and a Master (1991) in Electrical Engineering form KULeuven. His main research interests are ultra low power DSP and memories. Wim also is very active in the research towards a novel STEM education programs for the Flemisch secondary school system. He is a member of the ESSCIRC TPC and served on the ISSCC TPC for many years.

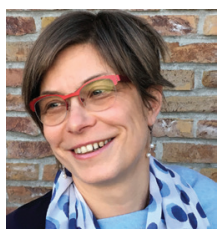

Mieke De Cock is professor in the Department of Physics and Astronomy, KU Leuven. She teaches introductory physics to first year students majoring in pharmaceutical sciences and is responsible for the physics teacher training program.Her major research interests are conceptual understanding and the interplay between mathematics and physics, both in secondary and higher education. She also is involved in research on integrated STEM education. 